\title{
PHOTOSYNTHETIC PHYSIOLOGICAL CHARACTERISTICS OF FOUR MEDICINAL PLANTS
}

\author{
MA, Y. $.^{1,2}-$ DING, Y. ${ }^{3}-$ LU, J..$^{*}$

 \\ ${ }^{2}$ College of Landscape Architecture, Changchun University, Changchun 130012, China \\ ${ }^{3}$ College of Art and Design, Jilin Jianzhu University, Changchun 130118, China \\ *Corresponding author \\ e-mail: ahappyday9217@126.com
}

(Received $9^{\text {th }}$ May 2019; accepted $16^{\text {th }}$ Jul 2019)

\begin{abstract}
The objective of this research was the study of research on the photosynthetic characteristics of four kinds of medicinal plants, to provide a reliable reference for the high quality cultivation of these four plants. Methods: the four kinds of medicinal plants were as the experimental materials. The photosynthetic rate, chlorophyll content, carotenoid content and photosynthesis of four medicinal plants were determined. The effects of net photosynthetic rate on photosynthetic effective radiation were studied, and the water use efficiency and transpiration rate were calculated. Results: through the comparison of net photosynthetic rate, it can be seen that the average photosynthetic rate, chlorophyll content and carotene content of the four medicinal plants are very different in the growing season. Conclusion: Platycodon grandiflorum has a wide range of adaptability to light intensity. It can not only utilize intense light efficiently, but also has shade tolerance. It is suggested to be cultivated in an intense light environment; North Atractylodes has high light utilization rate, but poor shade tolerance, it is suitable for cultivation in direct sunlight environment; Carthamustinctorious and Menthahaplocalyxhas have strong shade tolerance, but are not resistant to intense light, and can adopt intercropping.
\end{abstract}

Keywords: medicinal plants, photosynthetic rate, content of photosynthetic pigment, physiological and ecological factors, water use efficiency

\section{Introduction}

Traditional Chinese medicine is an important weapon in the prevention and treatment of diseases in Chinese traditional medicine. It has been playing an important role in protecting health. Medicinal plants contain bioactive ingredients, is used for special economic crops and anticorrosion treatment (Kannan et al., 2018). As an important industrial raw material, it can be processed into drugs, pesticides and veterinary drugs (Qadir et al., 2018; Khosravi et al., 2018). China is the country with the largest species of medicinal plants in the world, and has a long history of cultivation. Because medicinal plants are natural, stable, with little toxic and side effects, they have the unique advantages that Western medicine does not have. Modern people are increasingly turning their attention from synthetic chemicals to natural medicines, and hoping to reduce the incidence of drug-induced diseases (Liu et al., 2014).

With the vigorous development of the pharmaceutical industry, the establishment of plant research institutes has been established (Kim et al., 2018; Allahdadi et al., 2018). The production of Chinese herbal medicines has reached an unprecedented level from the variety and scale (Zhu et al., 2014). Unlike the field crop production, the economic characters such as yield and quality of Chinese herbal medicines are not easy to control, and their adaptability is poor. Moreover, because of the "absolute heterogeneity" of 
natural plants, the quality evaluation of Chinese herbal medicines is more difficult. Therefore, it is of great theoretical and practical significance (Huang et al., 2016) to study the fertility laws and various physiological characteristics of traditional Chinese medicine. At present, standardized construction of traditional Chinese medicine production bases, breeding and utilization of germplasm resources and excellent varieties, and pesticide residues and heavy metals are all actively studied. However, there are few reports about the relationship between photosynthetic performance and diurnal variation of photosynthesis and ecological factors, especially the research on main limiting factors and physiological characteristics of photosynthesis in different medicinal plants.

Photosynthesis is the process of transforming inorganic matter into organic matter and converting light energy into chemical energy. It is the basis of plant matter production (Lee et al., 2016). Photosynthetic rate determines the ability of photosynthesis, and is the key to the formation of plant biomass. The photosynthetic rate increases with the increase of light intensity in a certain range.

In this study, for further understanding of medicinal plant photosynthesis and physiological changes, the photosynthetic and physiological characteristics and environmental factors of four kinds of medicinal plants (Platycodon grandiflorum, North Atractylodes, Carthamustinctorious and Menthahaplocalyx) are determined and analyzed, and the diurnal changes of net photosynthetic rate and the content of photosynthetic pigments (Chlorophyll a, b, and carotenoid) were measured. Through light saturation point, light compensation point and photosynthetic pigment content, the shade tolerance and photosynthetic capacity were determined, the response of net photosynthetic rate to photosynthetically active radiation was discussed, the transpiration rate and water use efficiency were calculated as well as the physiological effects of ecological factors on the diurnal variation of net photosynthetic rate. The photosynthetic and physiological characteristics of these four medicinal plants are further studied to provide experimental basis for the creation of the cultivation environment which is beneficial to improve its photosynthesis.

\section{Materials and methods}

\section{Materials}

\section{Brief introduction of materials}

Platycodon grandiflorum: a perennial herb of the family Platycodon, and it grows in the shrubs region of less than $2100 \mathrm{~m}$ above sea level. It has the effects of relieving sore throat, and eliminating phlegm, etc. (Kim et al., 2016; Wanget al., 2015). Platycodon grandiflorum is not only a kind of resource plant but also a kind of economic plant for medicine and food. As shown in Figure 1 of Platycodon grandiflorum.

North Atractylodes: is a perennial herb of family Compositae, mainly divided into the North Atractylodes and the South Atractylodes. The North Atractylodes is mainly distributed in the north of China. It grows on the dry slopes of 300-900 m above sea level, with sparse broad-leaved forests or coniferous broad-leaved mixed forests (Gao et al., 2017; Shen et al., 2017). The site is the main use of Atractylodes rhizome, with eliminating heat and removing sweat, digestion and other effects, so the treatment used to ease bloating, diarrhea and edema disease, night blindness, rheumatism and diabetes and so on. As shown in Figure 2 of North Atractylodes. 


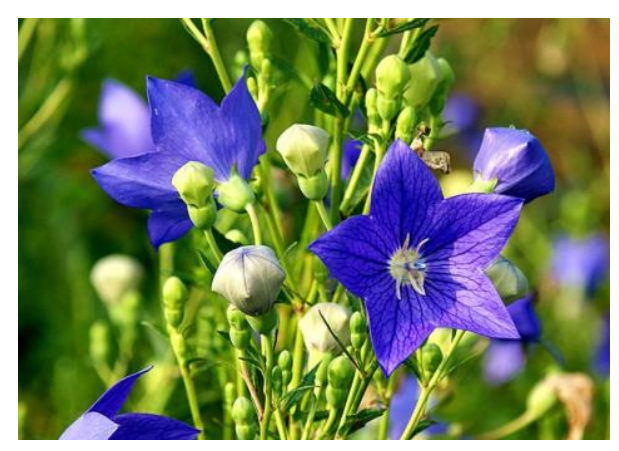

Figure 1. Platycodon grandiflorum

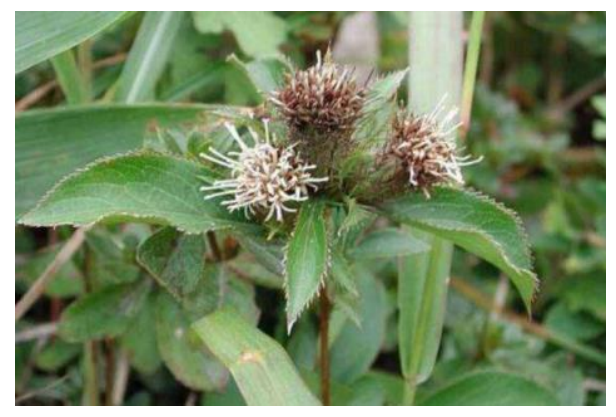

Figure 2. North Atractylodes

Carthamustinctorious: is an annual herbaceous plant of family Compositae. It has special fragrance, warm and spicy taste, it has the effects of activating blood circulation, removing blood stasis, dysmenorrhea and relieving pain. It also has the effects of antiinflammatory, improving myocardial blood supply, anti-aging and protecting liver. Safflower yellow is also a natural quality food pigment and dyestuff. As shown in Figure 3 of Carthamustinctorious.

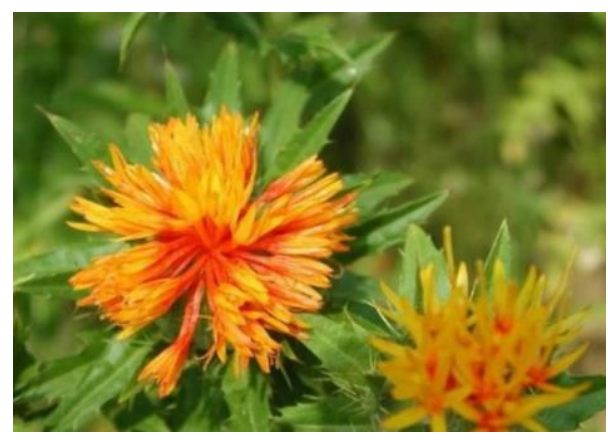

Figure 3. Carthamustinctorious

Menthahaplocalyx: is a perennial herb of the family Lamiaceae. Mint can grow in a region of less than $2100 \mathrm{~m}$ above sea level. It has the function of evacuating the wind and heat, clearing the head, and regulating the flow of vital energy and remove obstruction to it and relieving depression. It is good for preventing and curing cholesterol calculus, and has a role in central nervous system and digestive system. It is 
mainly for treating wind heat and common cold, headache and stuffiness, throat swelling and pain, rubella and itching. As shown in Figure 4 of Menthahaplocalyx.

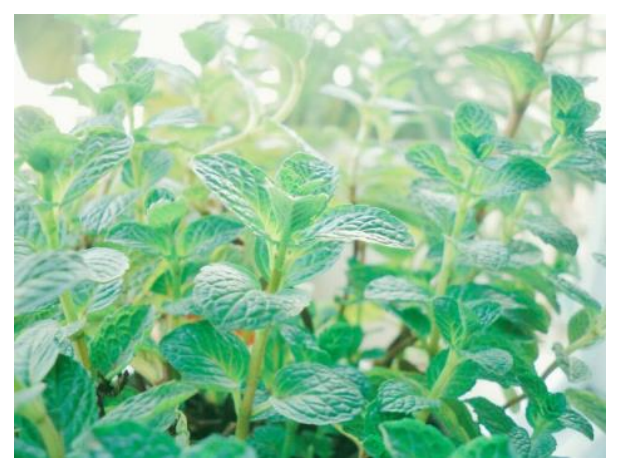

Figure 4. Menthahaplocalyx

\section{Experimental materials}

Study area survey: the test was carried out in the agricultural science and technology demonstration base of Tongzhou District Agricultural Technology Extension Station in Beijing. This area is a continental monsoon climate zone, the annual average temperature is $11.3{ }^{\circ} \mathrm{C}$, the annual rainfall is $620 \mathrm{~mm}$, and the annual sunshine is $2730 \mathrm{~h}$. The soil fertility is medium, and the texture is medium loam.

Experimental materials: Platycodon grandiflorum: in mid-April, the seeds of Platycodon grandiflorum, which were full of maturity, were seeded after germination in early May, the line spacing was $20 \mathrm{~cm} \times 20 \mathrm{~cm}$, and the routine field management measures were adopted. North Atractylodes: was collected from the Dabie Mountains in Hubei Province, which was identified by the Institute of traditional Chinese medicine of Hubei province. At the end of the March, Carthamustinctorious, Menthahaplocalyx were sowed in the experimental base, soil preparation, fertilizing, weeding, ridging and field management were carried out according to agronomic measures of common origin.

\section{Methods}

\section{Determination of photosynthetic rate of leaves}

From each cultivated plant population, a number of leaves were selected to accept the natural solar radiation. The gas exchange measurement (Nie et al., 2014) was carried out by a portable optical gas analysis system (LI-6400, Li-CorInc, Lincoln NE, USA). The control of the environmental factors in the leaf chamber was $20^{\circ} \mathrm{C}$, the concentration of the gas source $\mathrm{CO}_{2}$ was $350 \mu \mathrm{mol} \mathrm{CO}_{2}^{\circ} \mathrm{mol}^{-1}$, and the light flux density (PFD) was $1000 \mu \mathrm{mol}$ photo $n \mathrm{~s}^{\circ} \mathrm{m}^{-2} \mathrm{~s}^{-1}$. The experiment was carried out on all sunny days and repeated 5 6 times.

The calculation of the quantum efficiency ( $A Q Y$ ) of the leaves shows a linear regression of the net photosynthetic rate $\left(P_{n}\right)$ between $0 \sim 200 \mu \mathrm{mol}$ photo $n s^{\circ} m^{-2} s^{-1}$ in the response curve to $P F D$, and the regression equation is obtained.

$$
P_{n}=R_{d}+A Q Y \times P F D
$$


when $P_{n}=0, P F D$ is the optical compensation point $\Phi_{i}$ for photosynthesis. Where, $R_{d}$ is the rate of dark respiration.

The calculation of the carboxylation efficiency of the leaves was linear regression on the $0 \sim 200 \mu \mathrm{mol} \mathrm{CO}{ }_{2}^{\circ} \mathrm{mol}^{-1}$ point in the $\mathrm{C}_{i}$ curve of the intercellular $\mathrm{CO}_{2}$ concentration by $P_{n}$, and the regression equation was obtained.

$$
P_{n}=R_{p}+C E \times C_{i}
$$

when $P_{n}=0, C_{i}$ is the $\mathrm{CO}_{2}$ compensation point $\Gamma$ of photosynthesis. $R_{p}$ is the rate of respiration in the light, and $C E$ is the carboxylation efficiency. As dark breathing is very small, the rate of $\mathrm{CO}_{2}$ release can be approximated as the rate of photorespiration.

\section{Determination of chlorophyll content}

The leaves of the mature leaves with the diameter of $0.7 \mathrm{~cm}$ were collected from 30 mature leaves. The leaves were divided into 3 groups. They were immersed in the extract of $10 \mathrm{ml} 80 \%$ acetone. The sample bottle was tightly sealed, and it was colorless under low temperature. When the concentration of leaf green was detected, the concentration of chlorophyll was obtained by detecting the light intensity of the fluorescence (Ribeiro-Filho et al., 2014). Portable optical gas analysis system is designed based on the principle that different gases have selective absorption of light. It is often used to detect carbon monoxide, carbon dioxide and other gases. The formula for calculating the fluorescence intensity is:

$$
F=k Q I_{0}\left(1-e^{-\varepsilon c b}\right)
$$

in Equation 3: k is a constant; Q is the fluorescence efficiency; $I_{0}$ is the laser light intensity; $\mathrm{c}$ is the concentration of chlorophyll $\mathrm{a}$; $\mathrm{b}$ is the light path for the test sample; $\varepsilon$ is the molar absorbing coefficient.

In the way of logarithmic calculation, the formula is arranged and the logarithm is obtained.

$$
C=A-B \log (D-F)
$$

where $A=\frac{2.3}{\varepsilon b} \log k Q I_{0}, B=\frac{2.3}{\varepsilon b}, D=k Q I_{0}$.

$\mathrm{A}, \mathrm{B}, \mathrm{D}$ in Equation 4 are constant. When the sample of the fluorescence is determined, $\varepsilon, \mathrm{k}, \mathrm{Q}$, and $I_{0}$ are constant. Therefore, by measuring the fluorescence intensity of chlorophyll a emitted from cells, it can determine the concentration chlorophyll $\mathrm{a}$ and the fluorescence intensity of chlorophyll $\mathrm{b}$ to determine the concentration of chlorophyll $b$.

\section{Determination of carotenoid content}

The mixture of ethanol: acetone as 1:1 was used to extract $24 \mathrm{~h}$ in the dark, then be measured by UV-2001 ultraviolet spectrophotometer. And then the carotenoid content was calculated according to Equation 5. 


$$
C_{x}=\left(1000 D_{470}-2.05 C_{a}-114.8 C_{b}\right) / 245
$$

$D_{470}$ is the absorbance at the wavelength of $470 \mathrm{~nm}$. Pigment content $=($ pigment concentration $\times$ extract volume) / fresh weight of leaves.

Response of net photosynthetic rate to photosynthetically active radiation $\left(P_{n}-P A R\right)$

Using the red and blue light source, the light quantum flux density (PFD) in the Li6400 portable photosynthetic system were $0,400,800,1200,1600,2000$, $2400 \mu \mathrm{mol} \cdot \mathrm{m}^{-2} \cdot \mathrm{s}^{-1}$, respectively. The temperature was $27^{\circ} \mathrm{C}$ and the concentration of $\mathrm{CO}_{2}$ is set by $\mathrm{CO}_{2}$ injection system to $390 \mu \mathrm{mol} \cdot \mathrm{mol}^{-1}$, which was actually $390 \pm 10 \mu \mathrm{mol} \cdot \mathrm{mol}^{-1}$. The response of the net photosynthetic rate to the photosynthetic effective radiation was measured by the non-right angled hyperbolic model.

$$
P_{n}=\frac{a I=P_{\max }-\sqrt{\left(a I+P_{\max }\right)^{2}-4 a k I P_{\max }}}{2 k}-R_{d}
$$

According to the light response equation, the simulated photosynthetic response curve was estimated, the light compensation point (LCP) and the optical saturation point (LSP) were calculated. That is to say, when the $P_{n}$ reaches a certain level of $P_{\max }$, the $I$ value was obtained, and the light saturation point was estimated (Shen et al., 2016). The value of $I$ is used to estimate optical compensation point with $P_{n}$ at 0 . In Equation $6, P_{n}$ is the net photosynthetic rate $\left(\mu \mathrm{mol} \cdot \mathrm{m}^{-2} \cdot \mathrm{s}^{-1}\right), a$ is the use efficiency of optical quantum, $I$ is the light flux density $\left(\mu \mathrm{mol} \cdot \mathrm{m}^{-2} \cdot \mathrm{s}^{-1}\right), P_{\max }$ is the maximum total photosynthetic rate $\left(\mu \mathrm{mol} \cdot \mathrm{m}^{-2} \cdot \mathrm{s}^{-1}\right), R_{d}$ is dark respiration efficiency $\left(\mu \mathrm{mol} \cdot \mathrm{m}^{-2} \cdot \mathrm{s}^{-1}\right)$, and $k$ reflects the degree of curvature of the photosynthesis curve.

\section{Determination of photosynthesis and ecophysiological factors}

Li-6400 portable photosynthesis measurement system produced by LI-COR Company was used. The net photosynthetic rate $\left(P_{n}\right)$ of plant leaves was measured under natural condition. The time interval was 8:00-18:00, every $2 \mathrm{~h}$ was measured once, five values were recorded each time, and the average value was recorded. Li-6400 portable photosynthesis measurement system also recorded physiological and ecological factors such as temperature $\left(T_{a}\right)$, relative humidity $(\mathrm{RH})$, photosynthetically active radiation (PAR), stomatal conductance $\left(G_{s}\right)$ and transpiration rate E, etc. (Ding et al., 2015).

\section{Water use efficiency}

Water use efficiency $(\mathrm{WUE})=$ net photosynthesis rate $* 44 /$ transpiration rate $* 18$, unit: $m g \mathrm{CO}_{2} / \mathrm{gH}_{2} \mathrm{O}$.

\section{Results}

The Figure 5 is the leaves' photosynthetic rate comparison chart of four species of medicinal plants during the whole growth period. The Figure 6 is a daily change map of 
the environment factor. The Figure 7 is the effect of net photosynthetic rate on photosynthetically active radiation.

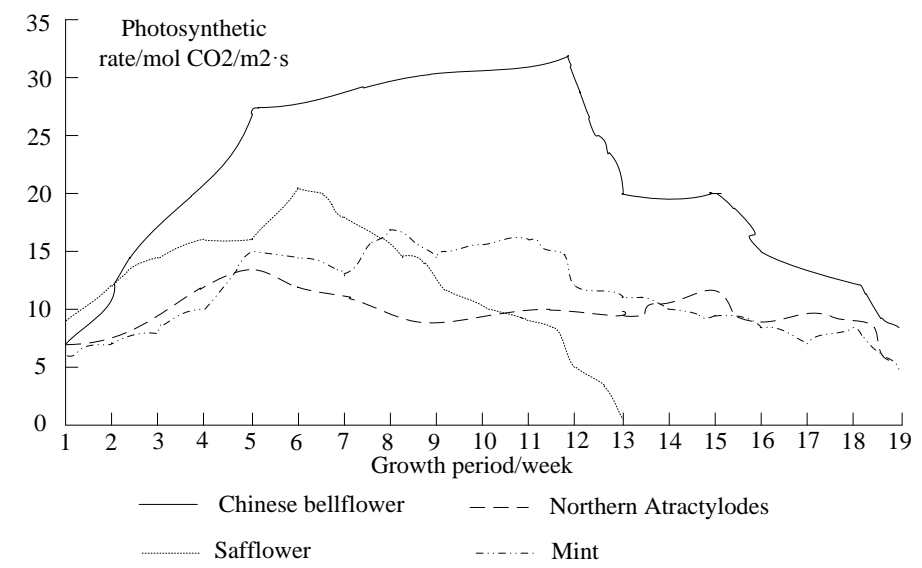

Figure 5. Contrast diagram of photosynthetic rate of leaves at full growth stage



Figure 6. Diurnal variation of environmental factors

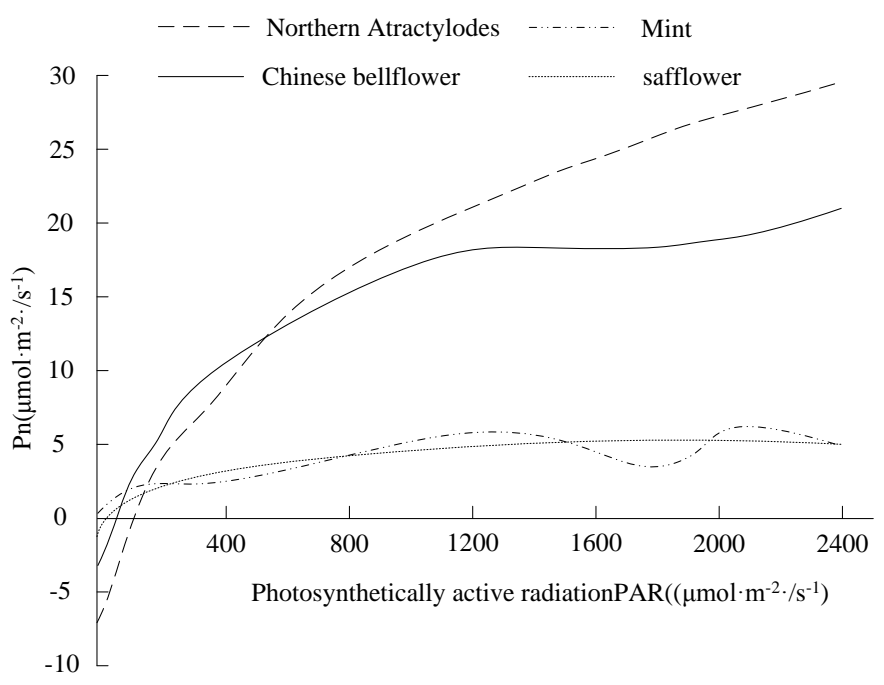

Figure 7. Effect of net photosynthetic rate on photosynthetically active radiation 
Table 1 is the photosynthetic parameters of four medicinal plants. Table 2 is the content of photosynthetic pigments in four medicinal plants. Table 3 is the path analysis between the main factors affecting the net photosynthetic rate.

Table 1. Photosynthetic parameters of 4 medicinal plants

\begin{tabular}{|c|c|c|c|}
\hline Medicinal plant & $\mathrm{LCP}\left(\mu \mathrm{mol} \cdot \mathrm{m}^{-2} \mathrm{~s}^{-1}\right)$ & LSP $\left(\mu \mathrm{mol} \cdot \mathrm{m}^{-2} \mathrm{~s}^{-1}\right)$ & $\operatorname{Pmax}\left(\mu \mathrm{mol} \cdot \mathrm{m}^{-2} \mathrm{~s}^{-1}\right)$ \\
\hline Platycodon grandiflorum & $45 \pm 6.03 \mathrm{~b}$ & $1052 \pm 113.16 b$ & $21.8 \pm 2.02 b$ \\
\hline North Atractylodes & $106 \pm 10.97 \mathrm{a}$ & $1558 \pm 162.97 \mathrm{a}$ & $29.7 \pm 2.86 \mathrm{a}$ \\
\hline Carthamustinctorious & $38 \pm 6.56 b$ & $307 \pm 42.67 \mathrm{c}$ & $5.8 \pm 0.76 \mathrm{c}$ \\
\hline Menthahaplocalyx & $7 \pm 1.53 \mathrm{c}$ & $261 \pm 12.17 \mathrm{c}$ & $5.0 \pm 0.53 \mathrm{c}$ \\
\hline
\end{tabular}

The difference between the different letters in the same column is significant $(\mathrm{p}<0.05)$, the same below

Table 2. Content of photosynthetic pigments in 4 medicinal plants

\begin{tabular}{c|c|c|c}
\hline Medicinal plant & Chlorophyll $\left(\mathbf{m g} \cdot \mathbf{g}^{-\mathbf{1}}\right)$ & Carotenoid $\left.\mathbf{( m g} \cdot \mathbf{g}^{\mathbf{- 1}}\right)$ & Chlorophyll a/b \\
\hline Platycodon grandiflorum & $1.769 \pm 0.0212 \mathrm{~b}$ & $0.342 \pm 0.0164 \mathrm{~b}$ & $2.880 \pm 0.0070 \mathrm{~b}$ \\
North Atractylodes & $1.085 \pm 0.0321 \mathrm{c}$ & $0.204 \pm 0.0151 \mathrm{c}$ & $3.189 \pm 0.4020 \mathrm{a}$ \\
Carthamustinctorious & $1.919 \pm 0.0617 \mathrm{a}$ & $0.417 \pm 0.0204 \mathrm{a}$ & $2.861 \pm 0.0863 \mathrm{~b}$ \\
Menthahaplocalyx & $1.701 \pm 0.1021 \mathrm{~b}$ & $0.396 \pm 0.0178 \mathrm{a}$ & $2.471 \pm 0.0732 \mathrm{c}$ \\
\hline
\end{tabular}

Table 3. Path analysis between the main factors affecting the net photosynthetic rate

\begin{tabular}{c|c|c|c|c|c|c|c|c}
\hline Medicinal plant & $\begin{array}{c}\text { Influence } \\
\text { factor }\end{array}$ & $\begin{array}{c}\text { Direct } \\
\text { action }\end{array}$ & $\begin{array}{c}\text { Indirect } \\
\text { effect of Ta }\end{array}$ & $\begin{array}{c}\text { Indirect } \\
\text { effect of RH }\end{array}$ & $\begin{array}{c}\text { Indirect effect } \\
\text { of PAR }\end{array}$ & $\begin{array}{c}\text { Indirect } \\
\text { effect of Gs }\end{array}$ & $\begin{array}{c}\text { Total } \\
\text { effect }\end{array}$ & $\begin{array}{c}\text { Decision } \\
\text { coefficient }\end{array}$ \\
\hline \multirow{5}{*}{ Platycodon grandiflorum } & Ta & -0.2472 & & -0.0016 & 0.2252 & 0.0201 & 0.1785 & -0.149380 \\
& RH & 0.0017 & 0.2208 & & -0.2307 & -0.2711 & -0.2793 & -0.000950 \\
& PAR & 0.3687 & -0.1509 & -0.0011 & & 0.4360 & $0.6527 * *$ & 0.345357 \\
& Gs & 0.8103 & -0.0616 & -0.0006 & 0.1984 & & $0.9465^{* *}$ & 0.877275 \\
\hline \multirow{5}{*}{ North Atractylodes } & Ta & -0.0008 & & -0.1081 & 0.2444 & 0.1049 & 0.2404 & -0.000390 \\
& RH & 0.1211 & 0.0008 & & -0.2325 & -0.2004 & -0.3110 & -0.090010 \\
& PAR & 0.3339 & -0.0006 & -0.0843 & & 0.5025 & $0.7515 * *$ & 0.390402 \\
& Gs & 0.7938 & -0.0001 & -0.0306 & 0.2114 & & $0.9745 * *$ & 0.917012 \\
\hline \multirow{5}{*}{ Marthamustinctorious } & Ta & -0.0642 & & -0.1344 & 0.0928 & -0.1308 & -0.2366 & 0.026247 \\
& RH & 0.1547 & 0.0557 & & -0.0619 & -0.1042 & 0.0443 & -0.010180 \\
& PAR & 0.1425 & -0.0418 & -0.0671 & & 0.2558 & 0.2894 & 0.062163 \\
& Gs & 0.9429 & 0.0089 & -0.0171 & 0.387 & & $0.9734 * *$ & 0.946522 \\
\hline
\end{tabular}

Figure 8 is the diurnal variation of the stomatal conductance and net photosynthetic rate. Figure 9 is a seasonal change in net photosynthetic rate.

Table 4 is the changes of transpiration rate (unit: $\mathrm{mg} / \mathrm{g} \mathrm{FW}$ ) of Platycodon grandiflorum and North Atractylodes in the whole growth period. Table 5 is the changes of transpiration rate (unit: $\mathrm{mg} / \mathrm{g} \mathrm{FW}$ ) of Carthamustinctorious and Menthahaplocalyx in the whole growth period. Table 6 is the water use efficiency of Platycodon 
grandiflorum and North Atractylodes in the whole growth period (unit $\mathrm{mgCO}_{2} / \mathrm{gH}_{2} \mathrm{O}$ ). Table 7 is the water use efficiency of Carthamustinctorious and Menthahaplocalyx in the whole growth period (unit $\mathrm{mgCO}_{2} / \mathrm{gH}_{2} \mathrm{O}$ ).

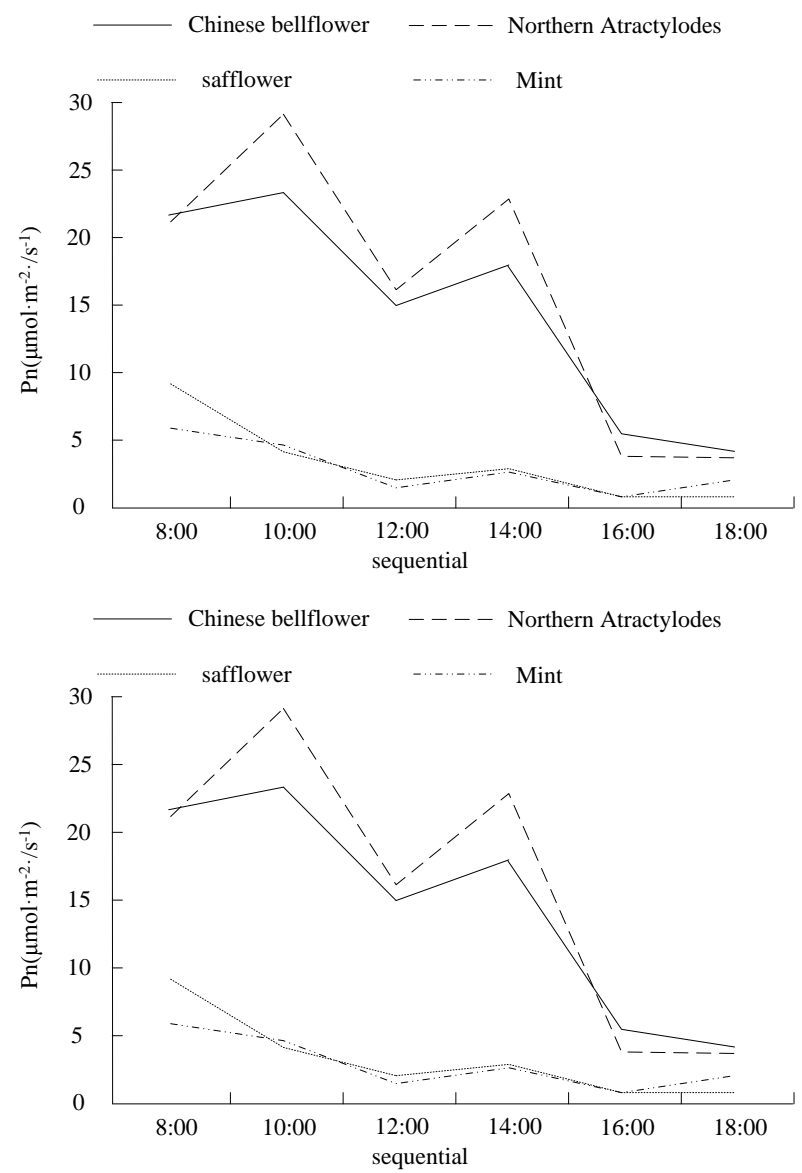

Figure 8. Diurnal variation of stomatal conductance and net photosynthetic rate

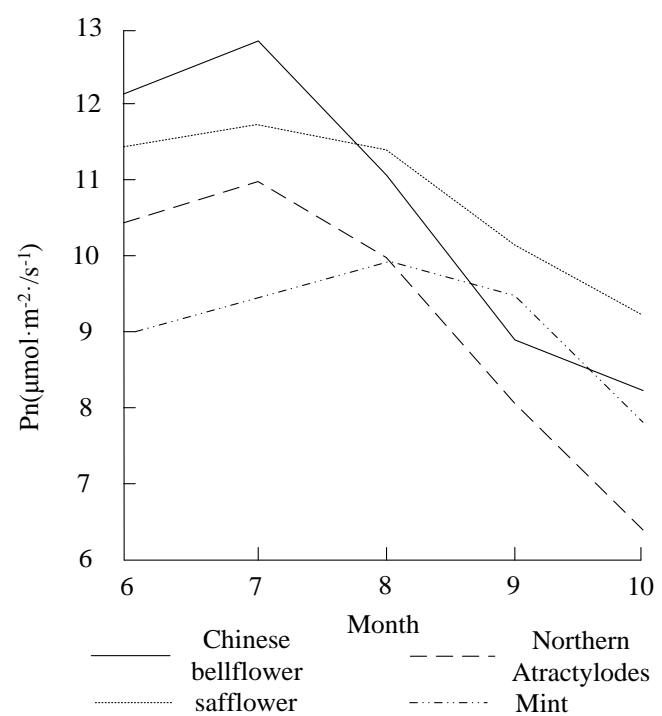

Figure 9. Seasonal variation of net photosynthetic rate 
Table 4. Change of transpiration rate of Platycodon grandiflorum and North Atractylodes during the whole growth period

\begin{tabular}{|c|c|c|c|c|c|}
\hline \multicolumn{3}{|c|}{ Platycodon grandiflorum } & \multicolumn{3}{|c|}{ North Atractylodes } \\
\hline Period of birth & Date & $\begin{array}{l}\text { Transpiration } \\
\text { rate } / \text { g }^{-2} \mathbf{m}^{-2} \cdot \mathbf{h}^{-1}\end{array}$ & Period of birth & Date & $\begin{array}{l}\text { Transpiration } \\
\text { rate } / g^{\circ} \mathbf{m}^{-2} \cdot \mathbf{h}^{-1}\end{array}$ \\
\hline \multirow{3}{*}{ Seedling stage } & $05-10$ & 1.50 & \multirow{3}{*}{ Seedling stage } & $05-10$ & 2.06 \\
\hline & $05-24$ & 1.66 & & $05-17$ & 2.04 \\
\hline & $06-07$ & 2.27 & & $05-24$ & 2.86 \\
\hline \multirow{3}{*}{ Rosette stage } & $06-14$ & 4.02 & \multirow{3}{*}{ Florescence } & $06-10$ & 3.81 \\
\hline & $06-22$ & 3.42 & & $06-22$ & 3.06 \\
\hline & 07-07 & 3.09 & & $07-01$ & 2.41 \\
\hline \multirow{3}{*}{ Florescence } & $07-25$ & 2.43 & \multirow{3}{*}{ Fruit } & $07-13$ & 2.33 \\
\hline & 08-05 & 2.54 & & $07-19$ & 2.33 \\
\hline & $08-16$ & 1.81 & & $07-25$ & 2.29 \\
\hline \multirow{5}{*}{ Fruit } & $08-31$ & 1.52 & \multirow{3}{*}{ Bulking period of tuber } & 08-05 & 2.78 \\
\hline & 09-10 & 1.27 & & $08-16$ & 2.90 \\
\hline & $09-26$ & 1.28 & & $08-31$ & 2.74 \\
\hline & & & \multirow{2}{*}{ Tuber weight gain period } & $09-10$ & 2.04 \\
\hline & & & & $09-25$ & 1.63 \\
\hline
\end{tabular}

Table 5. The change of transpiration rate in the whole growth period of Carthamustinctorious and Menthahaplocalyx

\begin{tabular}{|c|c|c|c|c|c|}
\hline \multicolumn{3}{|c|}{ Carthamustinctorious } & \multicolumn{3}{|c|}{ Menthahaplocalyx } \\
\hline Period of birth & Date & $\begin{array}{l}\text { Transpiration } \\
\text { rate } / \mathbf{g} \cdot \mathbf{m}^{-2} \cdot \mathbf{h}^{-1}\end{array}$ & Period of birth & Date & $\begin{array}{l}\text { Transpiration } \\
\text { rate } / \mathbf{g}^{\circ} \cdot \mathbf{m}^{-2} \cdot \mathbf{h}^{-1}\end{array}$ \\
\hline \multirow{3}{*}{ Rosette stage } & 5.10 & 1.52 & \multirow{3}{*}{ Seedling stage } & 5.10 & 1.43 \\
\hline & 5.17 & 2.66 & & 5.17 & 2.12 \\
\hline & 5.24 & 2.37 & & 5.24 & 2.65 \\
\hline \multirow{3}{*}{ Branching period } & 6.1 & 2.97 & \multirow{3}{*}{ Branching period } & 6.7 & 2.81 \\
\hline & 6.7 & 3.13 & & 6.14 & 2.40 \\
\hline & 6.14 & 3.43 & & 6.22 & 2.99 \\
\hline \multirow{3}{*}{ Florescence } & 6.22 & 2.75 & \multirow{3}{*}{ Florescence } & 7.7 & 2.91 \\
\hline & 7.1 & 2.32 & & 7.19 & 2.90 \\
\hline & 7.13 & 1.59 & & 7.25 & 2.44 \\
\hline \multirow{3}{*}{ Fruit } & 7.19 & 1.63 & \multirow{3}{*}{ Fruit } & 8.5 & 2.39 \\
\hline & 7.25 & 0.20 & & 8.16 & 2.09 \\
\hline & & & & 8.31 & 1.92 \\
\hline
\end{tabular}


Table 6. Water use efficiency of Platycodon grandiflorum and North Atractylodes at full growth stage

\begin{tabular}{c|c|c|c|c|c}
\hline \multicolumn{2}{c|}{ Platycodon grandiflorum } & \multicolumn{4}{c}{ North Atractylodes } \\
\hline \multirow{2}{*}{ Period of birth } & Date & $\begin{array}{c}\text { WUE } \\
(\mathbf{g} / \mathbf{k g})\end{array}$ & Period of birth & Date & $\begin{array}{c}\text { WUE } \\
(\mathbf{g} / \mathbf{k g})\end{array}$ \\
\hline \multirow{3}{*}{ Seedling stage } & 5.10 & 11.37 & & 5.10 & 2.06 \\
& 5.17 & 10.63 & Seedling stage & 5.17 & 2.04 \\
& 5.24 & 8.93 & & 5.24 & 2.86 \\
\hline \multirow{3}{*}{ Rosette stage } & 6.1 & 11.35 & & 6.11 & 3.81 \\
& 6.7 & 16.82 & Florescence & 6.22 & 3.06 \\
& 6.14 & 12.49 & & 7.1 & 2.41 \\
\hline \multirow{3}{*}{ Florescence } & 7.20 & 13.38 & & 7.13 & 2.33 \\
& 8.15 & 12.96 & & 7.19 & 2.33 \\
& 9.5 & 12.91 & & 7.25 & 2.29 \\
\hline \multirow{3}{*}{ Fruit } & 9.10 & 10.56 & & 8.5 & 2.78 \\
& 9.17 & 9.81 & Bulking period of tuber & 8.16 & 2.90 \\
& 9.25 & 8.02 & & 8.31 & 2.74 \\
\hline & & & & 9.10 & 2.04 \\
& & & Tuber weight gain period & 9.25 & 1.63 \\
\hline
\end{tabular}

Table 7. Water use efficiency of the full growth period of Carthamustinctorious and Menthahaplocalyx

\begin{tabular}{c|c|c|c|c|c}
\hline \multicolumn{3}{c|}{ Carthamustinctorious } & \multicolumn{3}{c}{ Menthahaplocalyx } \\
\hline Period of birth & Date & WUE & Period of birth & Date & WUE \\
\hline \multirow{3}{*}{ Rosette stage } & 5.10 & 12.39 & & 5.10 & 11.29 \\
& 5.17 & 11.66 & Seedling stage & 5.17 & 10.02 \\
& 5.24 & 14.84 & & 5.24 & 8.92 \\
\hline \multirow{3}{*}{ Branching period } & 6.1 & 12.88 & & 6.07 & 11.54 \\
& 6.7 & 12.78 & Branching period & 6.14 & 15.96 \\
& 6.14 & 13.79 & & 6.22 & 11.90 \\
\hline \multirow{3}{*}{ Florescence } & 6.22 & 15.89 & & 7.7 & 13.03 \\
& 7.1 & 16.45 & Florescence & 7.19 & 12.86 \\
& 7.13 & 16.92 & & 7.25 & 12.98 \\
\hline \multirow{2}{*}{ Fruit } & 7.19 & 11.56 & & 8.5 & 11.90 \\
& 7.25 & 7.57 & Fruit & 8.16 & 10.49 \\
& 7.31 & 5.42 & & 8.31 & 8.95 \\
\hline
\end{tabular}




\section{Data analysis}

The following results are derived from the experiments in the previous section.

\section{Comparison of photosynthetic rate in whole growth period}

From Figure 5, we can see that in the whole growth period, the net photosynthetic rate of medicinal plants propagated by seeds showed a single peak curve: that is, the seedling stage is low, and the net photosynthetic rate increases with the expansion of vegetative body. When it reached high in the period of flower and fruit, it was gradually reduced; while the change curve of net photosynthetic rate of the medical plants propagated by rhizome showed bimodal curve. This is because the physiological and biochemical characteristics of medicinal plants are also different. Therefore, there are some differences in net photosynthetic rate.

\section{The seasonal variation of net photosynthetic rate}

Figure 9 showed that plant leaves had matured in June, and the photosynthetic rate of the four medicinal plants began to rise, and the maximum of the growth season was reached about July. With the change of season, photosynthetic rate began to decrease. In October, photosynthetically active radiation and air temperature decreased, leaves began to senescence, physiological activity decreased, and photosynthetic rate decreased to the minimum value in growing season. In addition, four kinds of medicinal plant photosynthetic rate in the growing season was significantly higher than that at the end of the growing season, this was because the higher leaf physiological activity of plant growth season (Chen et al., 2015). But with the leaf senescence and seasonal variation of environmental factors, the physiological activity of leaves decreased, the efficiency of photosynthetic apparatus declined, and the ability of assimilating $\mathrm{CO}_{2}$ decreased, which showed that the photosynthetic rate of all plants decreased.

\section{Diurnal changes of environmental factors}

The environmental factors that affect plant photosynthesis include temperature, moisture, photosynthetic effective radiation, etc. The changes of temperature $\left(T_{a}\right)$, relative humidity $(\mathrm{RH})$ and photosynthetically active radiation (PAR) in the test day were shown in Figure 6.

In one day, the change of three environmental factors showed a single peak. PAR increased from $589 \mu \mathrm{mol} \cdot \mathrm{m}^{-2} \cdot \mathrm{s}^{-1}$ to $1989 \mu \mathrm{mol} \cdot \mathrm{m}^{-2} \cdot \mathrm{s}^{-1}$ in 8:00-14:00, and then dropped to a minimum at 18:00, only $91 \mu \mathrm{mol} \cdot \mathrm{m}^{-2} \cdot \mathrm{s}^{-1} . T_{a}$ was mainly affected by PAR $(\mathrm{r}=0899 * *)$, the largest was at $12: 00$, as $40.9{ }^{\circ} \mathrm{C}$, and to $22.3{ }^{\circ} \mathrm{C}$ at $18: 00$. RH and PAR was significantly negatively correlated with $T_{a}(\mathrm{p}<0.01)$. The correlation coefficient $r_{1}$ and $r_{2}$ were $-0.945^{* *} .-0.941 * *$, respectively.

\section{Diurnal variation of stomatal conductance $\left(G_{s}\right)$ and net photosynthetic rate $\left(P_{n}\right)$}

It can be clearly seen from Figure 8 that the changes of $G_{s}$ and $P_{n}$ of Platycodon grandiflorum and North Atractylodes were typical bimodal curve in one day, and the first peak > the second peak. This is due to the low light intensity and low temperature in the morning. At about 10:00, it reached the first peak, at this time PAR was $1161 \mu \mathrm{mol} \cdot \mathrm{m}^{-2} \cdot \mathrm{s}^{-1}$, and $T_{a}$ was $32{ }^{\circ} \mathrm{C}$. At about 12:00, Platycodon grandiflorum and North 
Atractylodes were in the "noon break" state of photosynthesis, $G_{s}$ decreased, and $P_{n}$ decreased. At about 14:00, $G_{s}$ and $P_{n}$ reached second peak. $G_{s}$ and $P_{n}$ of Carthamustinctorious and Menthahaplocalyx were the largest at 8:00, and they were at a lower level since then, and there was no obvious "noon break" phenomenon.

The daily change of net photosynthetic rate of Platycodon Platycodon and North Atractylodes was bimodal curve, and the "noon break" phenomenon of photosynthesis appeared. This is a kind of beneficial ecological adaptation and self-regulation mechanism for the hot summer in the plant.

\section{The relationship between the diurnal variation of net photosynthetic rate $\left(P_{n}\right)$ and ecophysiological factors}

The path analysis results of $P_{n}$ and eco physiological factors (Table 3) showed that $P_{n}$ and PAR of Platycodon grandiflorum and North Atractylodes was significantly positively correlated with $G_{s}$, but without correlation of $\mathrm{RH}$ and $T_{a} \cdot P_{n}$ of Carthamustinctorious and Menthahaplocalyx were positively correlated with $G_{s}$, not related to $\mathrm{RH}$ and $\mathrm{PAR}$, but negatively correlated with $T_{a}$. According to the physiological and ecological factors, the decision coefficient $P_{n}$ of the four medicinal plants in a day showed that the determinants that affected the diurnal variation of Platycodon grandiflorum and North Atractylodes were PAR and $G_{s}$, and PAR was the main determining factor. Limiting factors were $T_{a}$ and $\mathrm{RH}$, where $T_{a}$ was a major limiting factor for the diurnal variation of Carthamustinctorious $P_{n}$, and the limiting effect of $T_{a}$ and RH on North Atractylodes $P_{n}$ was small. $G_{s}$ was the main determinants of the diurnal variation of Carthamustinctorious, $T_{a}$ and PAR also had certain promoting effect on $P_{n}$, but the effect was very small; $\mathrm{RH}$ was a limiting factor of diurnal variation of Carthamustinctorious $P_{n} \cdot T_{a}, \mathrm{RH}, \mathrm{PAR}$ and $G_{s}$ were the limiting factors of the diurnal variation of Menthahaplocalyx $P_{n}$, and $T_{a}$ of them were the most important limiting factor.

\section{Comparison of photosynthetic parameters}

The light compensation point (LCP), the light saturation point (LSP) and the maximum net photosynthetic rate can directly reflect the tolerance and photosynthetic capacity of plants. The low LCP of plants means that plants have strong ability to use weak light. The low LSP showed that the photosynthetic rate of plants increased rapidly with the increase of light quantum density, and quickly reached the maximum efficiency. Therefore, lower LCP and LSP enable plants to maximize photosynthetic capacity under low light density and maximize their photosynthesis under light limitation, thereby enhancing the accumulation of organic matter and meeting their energy needs for survival and growth.

It can be seen from the light response of $P_{n}$ (Fig. 7) that the order of respiration rate of four medicinal plants were: Menthahaplocalyx $<$ Carthamustinctorious $<$ Platycodon grandiflorum $<$ North Atractylodes. In the PAR range of $0 \sim 100 \mu \mathrm{mol} \cdot \mathrm{m}^{-2} \cdot \mathrm{s}^{-1}$, the $P_{n}$ and PAR of four kinds of medicinal plants was closed tothe linear relationship. The $P_{n}$ of Menthahaplocalyx in the range wasthe maximum and that of North Atractylodes was the minimum. $P_{n}$ of Carthamustinctorious and Menthahaplocalyx increased slowly in 
that PAR was $100 \sim 300 \mu \mathrm{mol} \cdot \mathrm{m}^{-2} \cdot \mathrm{s}^{-1}$ and reached saturation, and then tended to be stable after $300 \mu \mathrm{mol} \cdot \mathrm{m}^{-2} \cdot \mathrm{s}^{-1} \cdot P_{n}$ of Platycodon grandiflorum and North Atractylodes increased rapidly in that PAR was $100 \sim 1000 \mu \mathrm{mol} \cdot \mathrm{m}^{-2} \cdot \mathrm{s}^{-1}$, and closed to saturation in about $1000 \mu \mathrm{mol} \cdot \mathrm{m}^{-2} \cdot \mathrm{s}^{-1}$ and $1600 \mu \mathrm{mol} \cdot \mathrm{m}^{-2} \cdot \mathrm{s}^{-1}$. The light compensation curve (LCP), light saturation point (LSP) and maximum net photosynthetic rate of the four medicinal plants were estimated according to the fitting equation of light response curve, shown in Table 3.

The results showed that the LCP, LSP and the maximum net photosynthetic rate of the North Atractylodes were significantly higher than those of the other 3 medicinal plants, indicating that they had strong ability to use intense light and high photosynthetic capacity under intense light, and $106 \mu \mathrm{mol} \cdot \mathrm{m}^{-2} \cdot \mathrm{s}^{-1}$ of LCP showed their shade tolerance. The LCP of Carthamustinctorious and Menthahaplocalyx was lower than that of the North Atractylodes, indicating that they had high ability to use weak light and strong shade tolerance. The LCP of Platycodon grandiflorum was close to Carthamustinctorious, while LSP was higher, and has a higher maximum net photosynthetic rate, indicating that its photosynthesis was effective and its adaptability to light was the largest. The maximum net photosynthetic rate of Carthamustinctorious and Menthahaplocalyx was significantly lower than that of Platycodon grandiflorum and North Atractylodes, while Platycodon grandiflorum was lower than that of North Atractylodes.

\section{Water use efficiency in the production of photosynthetic substances in medicinal plants}

It can be seen from Tables 6 and 7 that in the 4 medicinal plants, except the North Atractylodes, the remaining plants have a common characteristic in the water use efficiency, that is, the single peak curve changes in the whole growth period.

Differences between species in water use efficiency: Although the change of water use efficiency during the growth period is a single peak curve, for different plant species, water use efficiency has the characteristics of species, mainly in the following areas:

Firstly, the comparison of the whole growth period, Platycodon Grandiflorum has the highest water use efficiency of $15.62 \sim 23.22 \mathrm{mgCO} / \mathrm{gH}_{2} \mathrm{O}$, followed by Carthamustinctorious, Menthahaplocalyx, and the lowest is the North Atractylodes.

Secondly, the period of highest water use efficiency has interspecific difference: Platycodon grandiflorum, Carthamustinctorious and Menthahaplocalyx propagated by seeds is at flowering stage, while the North Atractylodes propagated by vegetative is in accumulation stage.

Thirdly, the water use efficiency of Platycodon grandiflorum is relatively stable during the whole growth period, that is, there is little difference in the growth period. For example, the water use efficiency of Platycodon grandiflorum at different growth stages was $7.21 \%-11.63 \%$ compared with the water use efficiency of the whole growth period, while the differences in the three other medicinal plants was more than $50 \%$.

\section{Change of transpiration rate of medicinal plants in the whole growth period}

Transpiration is the process of loss of water from the surface of the living plant (mainly leaves) in the state of water vapor into the atmosphere. It is different from the 
evaporation process of physics. Transpiration is not only influenced by environmental conditions, but also regulated and controlled by the plant itself, so it is a complex physiological process.

Transpiration is of great importance to plants, providing the main motive force for the plant to absorb and transport water, and also to lose the water. Photosynthesis is a way for plants to produce organic matter by means of light energy. It is often related to the importance of plant growth and crop yield.

The results of Tables 4 and 5 showed that the change of transpiration rate of different medicinal plants was similar to that of their net photosynthetic rate. $\circ$ The total change trend of transpiration rate in the whole growth period of the medicinal plants propagated by seeds showed a single peak curve: that is, the seedling stage was low, and transpiration rate gradually increased with the continuous expansion of the vegetative body. After reaching the highest level, it gradually reduced to the lowest in the late growth stage, and the transpiration rate of Platycodon grandiflorum varied greatly, While the North Atractylodes, which propagated with rhizomes and tubers, showed the bimodal curve.

There are some differences between the transpiration rates of medicinal plants: the Platycodon grandiflorum has the maximum transpiration rate, especially in the branching stage and the early flowering stage, between $2.43 \sim 4.02 \mathrm{~m} \mathrm{~mol} \mathrm{H}_{2} \mathrm{O} / \mathrm{m}^{2} / \mathrm{s}$, followed by Carthamustinctorious and Menthahaplocalyx, and the lowest is Atractylodis sinensis. The changes of the whole growth period of the Atractylodis sinensis is more stable.

\section{Discussion}

Photosynthesis is the process of green plant uses visible light energy to synthetize the carbon dioxide and hydration to become carbohydrate (usually glucose) through the chloroplast, and release oxygen ( $\mathrm{Zhu}$ et al., 2016). The reaction equation of

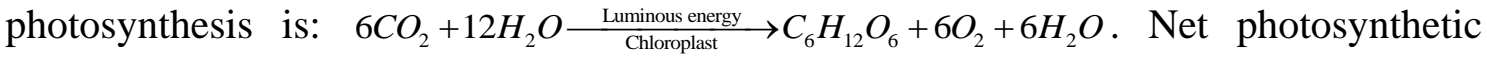
rate is closely related to its own factors, such as chlorophyll content, leaf thickness and leaf maturity. It is also affected by light intensity, air temperature, air relative humidity and soil water content (Schwinghamer et al., 2015).

\section{Analysis of photosynthetic rate during the whole growth period}

Among the four studied medicinal plants, Platycodon grandiflorum should be $\mathrm{C} 4$ plant and the other three species of medicinal plants are C3 plants. In each growth period, the net photosynthetic rate of Platycodon grandiflorum was significantly higher than that of North Atractylodes, Carthamustinctorious, Menthahaplocalyx cultivated in the same medicine Park. This is due to the $\mathrm{C} 4$ pathway of Platycodon grandiflorum, which has a fixed $\mathrm{CO}_{2}$, and the source of energy is continuously supplied and fully utilized by $\mathrm{CO}_{2}$, so it has a higher net photosynthetic rate (Schwinghamer et al., 2015).

The results showed that the photosynthetic rate of Carthamustinctorious and Menthahaplocalyx was slightly lower than that of North Atractylodes in the early stage of growth, while in the middle growth stage, it was significantly higher than that of North Atractylodes. The reason may be related to the difference of propagation mode (Peng et al., 2015): rhizome was used as propagating material for North Atractylodes, and soon entered fast growing stage, which showed strong photosynthetic ability. In 
addition, the time when the North Atractylodes entered the flowering and fruiting stage was earlier than that of Carthamustinctorious and Menthahaplocalyx, and there was a strong physiological bank.

In the middle growth period, the rapid growth of Carthamustinctorious and Menthahaplocalyx needs large amounts of organic material for the vegetative growth (Almeida et al., 2014), and although Atractylodes sinensis at this time also began to rapid growth of vegetative, because the strain was smaller and the consumption of organic substances were also relatively less. The net photosynthetic rate of the former is not high.

The difference in the medicinal parts was different: rhizome was the storage location of North Atractylodes, and it had swelling and nutrient accumulation in the late growth stage (Kjaer et al., 2014), thus showing another peak of net photosynthetic rate.

\section{The seasonal variation of net photosynthetic rate is discussed}

In June to July, the ability of Platycodon grandiflorumroot, Carthamustinctorious and North Atractylodes to use high temperature and high light intensity was obviously better than that of Menthahaplocalyx; In August and September, the photosynthetic capacity of Menthahaplocalyx increased slightly, indicating that Menthahaplocalyx could make use of relatively low light and temperature conditions more effectively. The photosynthetic rate of the North Atractylodes was the lowest in September and October, indicating that its ability to use relatively low light and temperature conditions was the lowest in four medicinal plants. The results showed that in the growing season, the order of average photosynthetic rate of four species of medicinal plants was: Platycodon grandiflorum $>$ Carthamustinctorious $>$ Menthahaplocalyx $>$ North Atractylodes.

\section{The diurnal variations of stomatal conductance and net photosynthetic rate are discussed}

The plant self-factors that cause the net photosynthetic rate of plant leaves decreased at noon including stomatal and non-stomatal factors. At noon, in the field, the intense light, high temperature, low humidity and other environmental factors caused by plant stomatal closures, $\mathrm{CO}_{2}$ was blocked to enter into the leaves and photosynthesis decreased, while non-stomatal factor was because the temperature was too high, resulting in a decline in net photosynthesis in mesophyll cells. In addition, under high light intensity for a long time, light inhibition and photorespiration can also lead to a decrease in net photosynthetic rate at noon. The response of $P_{n}$ from Platycodon grandiflorum and North Atractylodes to PAR showed that Platycodon grandiflorum and North Atractylodes still had high $P_{n}$ (above 19.5 and 26, $26.6 \mu \mathrm{mol} \cdot \mathrm{m}^{-2} \cdot \mathrm{s}^{-1}$ ) under intense light (above $1800 \mu \mathrm{mol} \cdot \mathrm{m}^{-2} \cdot \mathrm{s}^{-1}$ ), so photoinhibition and photorespiration were not the factors limiting $P_{n}$ at this time. At 12:00, RH dropped to the lowest point of the day, and $G_{s}$ was also lower than 10:00 and 14: 00, so the stomatal factor was one of the reasons for the low net photosynthetic rate at this time. In addition, $T_{a}$ reached $40.9{ }^{\circ} \mathrm{C}$ at this time. Previous studies found that the optimum activation temperature for the key enzyme of Rubisco in photosynthesis was at $25 \sim 30{ }^{\circ} \mathrm{C}$, its activity directly affects the photosynthetic rate. When the leaf temperature is too high, it will affect the activities of some related enzymes in the body, which is not conducive to the photosynthesis. 
Therefore, the decline of $P_{n}$ in the two plants is also caused by the decrease of the nonstomatal factors, the activity of the carboxycarboxylase of mesophyll cells.

The net photosynthetic rate of Carthamustinctorious and Menthahaplocalyx had a diurnal variation. The maximum net photosynthetic rate of the two plants was $9.1 \mu \mathrm{mol} \cdot \mathrm{m}^{-2} \cdot \mathrm{s}^{-1}$ and $5.8 \mu \mathrm{mol} \cdot \mathrm{m}^{-2} \cdot \mathrm{s}^{-1}$ respectively. After that, the net photosynthetic rate declined rapidly, which was similar to the results of the diurnal variation of the photosynthesis by Zhang Jingguang, et al. In the response of the $P_{n}$ of the two plants to the PAR, the $P_{n}$ decreased at PAR $>1400 \mu \mathrm{mol} \cdot \mathrm{m}^{-2} \cdot \mathrm{s}^{-1}$, indicating that the photoinhibition might have occurred under the intense light. The correlation analysis between diurnal variation of photosynthesis and physiological and ecological factors showed that $G_{s}$ is the main influence factor of Carthamustinctorious $P_{n}$, while $G_{s}$ of the Carthamustinctorious is low in a day, so stomatal limitation is a cause of low $P_{n}$ in the daytime. It is believed that high temperature and intense light cause the potential activity of the active center and the primary light conversion efficiency decreasing, which hindered the photosynthetic electron transport. It is the limiting factor of photosynthesis (Zhang et al., 2015).

\section{Content of photosynthetic pigment}

The pigment that absorbs light in photosynthesis is called photosynthetic pigment, and there are two main types in higher plants: chlorophyll (including chlorophyll a, b) and carotenoids. The color of the leaves is mainly a comprehensive representation of the proportion of green chlorophyll and yellow carotenoid. The chlorophyll $a / b$ of the leaves of the common sun plants is about $3: 1$, and the chlorophyll $a / b$ of the shade plants is $2: 3: 1$. The relative increase of chlorophyll $b$ content may make more effective use of the blue violet light in the leaching light. So the chlorophyll $b$ has the name of the chromophore system. The chlorophyll content of plants depends not only on site conditions, but also on the characteristics of plant species, and determines the ability of plants to absorb and convert light energy (Li et al., 2016).

The content of chlorophyll is also related to the resistance of plants to the shade. The chlorophyll content of Platycodon grandiflorum and Menthahaplocalyx had not significantly different, but the LSP and the maximum net photosynthetic rate of Platycodon grandiflorum were significantly higher than that of Menthahaplocalyx. The chlorophyll content of the North Atractylodes was significantly lower than that of Platycodon grandiflorum, but LSP was significantly higher than that of Platycodon grandiflorum, and the maximum net photosynthetic rate of them had no significant difference. The results showed that the content of chlorophyll was not directly related to LSP and the maximum net photosynthetic rate. The LCP of Platycodon grandiflorum, Carthamustinctorious and Menthahaplocalyx were lower than that of the North Atractylodes, so does the chlorophyll a/b value, but the content of chlorophyll and carotenoid was higher than that of the North Atractylodes, this may be because the plants with high chlorophyll content have weak absorption ability of light, it has better resistance to shade. In addition, it was found that low chlorophyll $\mathrm{a} / \mathrm{b}$ value increased the absorption of far red light. Therefore, plants with low chlorophyll a/b value also had higher photosynthetic activity under low intensity of light.

In the experiment, the chlorophyll content of Carthamustinctorious was the highest in the four medicinal plants, followed by Platycodon grandiflorum and 
Menthahaplocalyx, and that of North Atractylodes was the lowest. However, the content of chlorophyll a/b of North Atractylodes's is the highest, followed by Platycodon grandiflorum and Carthamustinctorious, and that of Menthahaplocalyx leaves is the lowest. Carthamustinctorious and Menthahaplocalyx in four medicinal plants have the highest carotenoid content.

\section{Conclusions}

With the continuous progress of the social development and the accelerated process of industrialization, agriculture and green land area is decreased. The study of Photosynthetic Physiology plays a very important role in the selection of germplasm resources, the rational allocation of planting environment and the increase of yield in medicinal plants (Niakan et al., 2015). According to the first two chapters, we can further understand their photosynthetic and physiological characteristics by studying and discussing four kinds of medicinal plants of Platycodon grandiflorum, Atractylodis sinensis, Carthamustinctorious and Menthahaplocalyx.

The Atractylodis sinensis has high utilization rate of intense light, but it belongs to the light plant, and has poor shade tolerance. Thus, it should be cultivated in the environment of direct sunlight enough to increase the yield. Carthamustinctorious and Menthahaplocalyx are shade loving plants, but they are not resistant to intense light. The cultivation environment outside the forest is easy to inhibit their photosynthesis. Therefore, the intercropping way of forest and medical plants can be adopted. Platycodon grandiflorum has great adaptability to light intensity. It can not only efficiently use intense light but also have a characteristic of certain shade tolerance. If it wants to increase photosynthetic rate and increase yield, it is recommended to cultivate it in an intense light environment.

In the future research stage, the ecological characteristics of the same species of medicinal plants in different growing areas will be studied in depth, so as to provide more theoretical support for the development and utilization of medicinal plants.

Acknowledgements. This work was supported by Jilin Science and Technology Development Plan Project - Demonstration of Large Scale Breeding and Cultivation Techniques of Chinese Medicinal Herbs, such as Parasenecio firmus (Komar.) Y. L. Chen and Platycodon grandiflorus (Jacq.) A. DC., Changbai Mountain (No. 20170307027YY).

\section{REFERENCES}

[1] Allahdadi, M. N., Li, C. (2018): Numerical simulation of Louisiana shelf circulation under Hurricane Katrina. - Journal of Coastal Research 34(1): 67-80.

[2] Almeida, A. A. F., Gomes, F. P., Araujo, R. P. (2014): Leaf gas exchange in species of the Theobroma, genus. - Photosynthetica 52(1): 16-21.

[3] Chen, G., Chen, B., Gao, S. (2015): Parallel simulation model for plant growth by integrating morphology and physiology. - Computer Simulation 32(8): 404-408.

[4] Ding, H., Yin, Q., Wan, G. (2015): Solubilization of menthol by platycodin D in aqueous solution: an integrated study of classical experiments and dissipative particle dynamics simulation. - International Journal of Pharmaceutics 480(2): 143-51. 
[5] Gao, W., Baig, A. Q., Ali, H., Sajjad, W., Farahani, M. R. (2017): Margin based ontology sparse vector learning algorithm and applied in biology science. - Saudi Journal of Biological Sciences 24(1): 132-138.

[6] Huang, Y. H., Jung, D. W., Lee, O. H. (2016): Fermented Platycodon grandiflorum extract inhibits lipid accumulation in 3T3-L1 adipocytes and high-fat diet-induced obese mice. - Journal of Medicinal Food 19(11): 1004.

[7] Kannan, P., Jithinraj, P., Natesan, M. (2018): Multiphasic inhibition of mild steel corrosion in H2S gas environment. - Arabian Journal of Chemistry 11(3): 388-404.

[8] Khosravi, A., Koury, R. N. N., Machado, L., Pabon, J. J. G. (2018): Prediction of hourly solar radiation in Abu Musa Island using machine learning algorithms. - Journal of Cleaner Production 176: 63-75.

[9] Kim, E. B., Nam, Y. H., Kang, T. H. (2016): Anti-diabetic activity of flavonoids isolated from the aerial parts of Platycodon grandiflorum in zebrafish, a model of type 2 diabetes. - Planta Medica 81(S 01): S1.

[10] Kim, N. Y., Jeon, E. J., Jung, S. H., Ahn, S. J., Park, M. A., Seo, J. S. (2018): Gene expression profiling and expression analysis of freshwater shrimp (Neocaridina denticulata denticulata) using expressed sequence tags and short-term exposure to copper. - Journal of Environmental Biology 39(1): 51-57.

[11] Kjaer, K. H., Clausen, M. R., Sundekilde, U. K. (2014): Photoperiodic variations induce shifts in the leaf metabolic profile of Chrysanthemum morifolium. - Functional Plant Biology 41(12): 1310-1322.

[12] Lee, N. K., Nyakudya, E., Jeong, Y. (2016): Bioconversion of platycodon Grandiflorum saponins by the platycodin D-converting microorganism, yeast Cyberlindnera Fabianii. Journal of Food Biochemistry 40(3): 358-365.

[13] Li, Z., Bai, W., Zhang, L. (2016): Increased water supply promotes photosynthesis, C/N ratio, and plantamajoside accumulation in the medicinal plant Plantago depressa, Willd. Photosynthetica 54(4): 1-9.

[14] Liu, M., Xu, Z., Guo, S. (2014): Evaluation of leaf morphology, structure and biochemical substance of balloon flower (Platycodon grandiflorum, (Jacq.) A. DC.) plantlets in vitro, under different light spectra. - Scientia Horticulturae 174(1): 112-118.

[15] Niakan, M., Mahjoob, S. R., Ghorbanli, M. (2015): Effect of exogenous putrescine on growth, photosynthesis and alkaloid compounds of Datura (Daturastramonium L.) in response to salinity stress under hydroponic conditions. - Journal of the Electrochemical Society 159(10): A1682-A1689.

[16] Nie, C., Liu, R., Li, S. (2014): Assessment of Platycodon grandiflorum germplasm resources from northern Anhui province based on ISSR analysis. - Molecular Biology Reports 41(12): 8195-201.

[17] Peng, H., Zhang, F., Jiang, J. (2015): Identification of quantitative trait loci for branching traits of spray cut chrysanthemum. - Euphytica 202(3): 385-392.

[18] Qadir, M. I., Mushtaq, H., Mobeen, T. (2018): In-silico study of potential carboxylic acid derivatives as D-glutamate ligase inhibitors in Salmonella typhi. - Kuwait Journal of Science 45(1): 100-107.

[19] Ribeiro-Filho, J., Leite, F. C., Costa, H. F. (2014): Curine inhibits mast cell-dependent responses in mice. - Journal of Ethnopharmacology 155(2): 1118-1124.

[20] Schwinghamer, T., Souleimanov, A., Dutilleul, P. (2015): The plant growth regulator lipo-chitooligosaccharide (LCO) enhances the germination of canola (Brassicanapus, [L.]). - Journal of Plant Growth Regulation 34(1): 183-195.

[21] Shen, W., Guan, Y., Wang, J. (2016): A polysaccharide from pumpkin induces apoptosis of HepG2 cells by activation of mitochondrial pathway. - Tumor Biology 37(4): 52395245 .

[22] Shen, Y., Mi, W., Zhang, Z. (2017): A positioning lockholes of container corner castings method based on image recognition. - Polish Maritime Research 24(SI): 95-101. 
[23] Wang, Y., Lu, B. H., Yang, L. N. (2015): First report of Fusarium armeniacum causing stem and root rot on Platycodon grandiflorus in Jilin Province, China. - Plant Disease 99(11): 150603065432001.

[24] Zhang, L. X., Guo, Q. S., Chang, Q. S. (2015): Chloroplast ultrastructure, photosynthesis and accumulation of secondary metabolites in Glechoma longituba, in response to irradiance. - Photosynthetica 53(1): 144-153.

[25] Zhu, L. X., Liu, D. (2014): Effects of different phosphorus applications on growth and platycodin content of Balloon Flower, (Platycodon grandiflorum). - Scientia Horticulturae 178(178): 8-13.

[26] Zhu, L. X., Zhang, W. J. (2016): Effects of controlled-release urea combined with conventional urea on nitrogen uptake, root yield, and quality of Platycodon grandiflorum. - Journal of Plant Nutrition 40(5): 662-672. 